2019-02-05

"You don't want to peer over people's

shoulders, it feels too rude!": the moral

geographies of using participants'

personal smartphones in research

Holton, Mark

http://hdl.handle.net/10026.1/10718

10.1111/area.12425

Area

Wiley

All content in PEARL is protected by copyright law. Author manuscripts are made available in accordance with publisher policies. Please cite only the published version using the details provided on the item record or document. In the absence of an open licence (e.g. Creative Commons), permissions for further reuse of content should be sought from the publisher or author. 


\section{"You don't want to peer over people's shoulders, it feels too rude!": the moral geographies of using participants' personal smartphones in research}

Holton, Mark (2018) - School of Geography, Earth, and Environmental Sciences, Plymouth University

Harmer, Nichola (2018) - School of Geography, Earth, and Environmental Sciences, Plymouth University

Accepted - 14 December 2017 (embargo until 14 December 2019)

Cite this article: Holton, M. and Harmer, N. (2018). "You don't want to peer over people's shoulders, it feels too rude!": the moral geographies of using participants' personal smartphones in research, Area. DOI:10.1111/area.12425

\section{Abstract}

This paper examines the moralities of using participants' personal mobile technologies in research. While smartphones bring novel benefits to research in terms of their labour-saving, innovative, intuitive and low-cost virtues, they also raise unique spatial, ethical and moral issues that can have implications for how research is conducted. Using morality as a conceptual tool this paper explores the smartphone as a geographical space that is simultaneously private/public, personal/shared and material/imagined. By examining the observations made during a pilot study which tested a mobile walking tour app, this paper questions the moral implications for, and the consequences of, researching using participants' personal technologies and how researchers might access and interpret behaviours within these private digital 'spaces'. This paper advocates the importance of recognising the complexities of moving into participants' private digital spaces and how a diverse range of behaviours may become part of the research encounter through the ways in which participants form interactions with technologies, the environment and with each other. Finally, this paper contributes to discussions of moral geographies by examining the asymmetric power relations that are evident when examining moral 
behaviours that are performed simultaneously in digital and geographical spaces, and questions whether these may be functions of hegemonic systems of 'acceptable' behaviour.

\section{Keywords}

Moral geographies; qualitative research; practice; methods; virtual space; smartphones

\section{Introduction}

There has been a recent drive to adopt emergent mobile technologies, such as smartphones and tablets, into contemporary geographical research practice (DeLyser and Sui, 2013; Gorman, 2017), both in terms of self-directed research (Neff and Nafus, 2016) and research[ing] with others (Brown et al., 2013; Laurier et al., 2016). Yet while advocates argue for the benefits of mobile technologies in generating understandings of, among other things, how people relate to technology, how technology might affect relationships with places and how researchers might capture 'new' data in real time, the ethics and moralities of adopting technologies into research remains unclear. While technologies add unique spatial dimensions to the qualitative methodological canon, this paper will demonstrate how technologies bring equally unique spatial, ethical and moral issues to research practice in ways that can seem innocuous at first but may have implications for how research is conducted, as well as how researchers interact with participants and work within digital 'spaces' that are not clearly delineated.

A key driver for these processes has been the adoption of personal mobile technologies that save time, resources, expert knowledge and costs. The 
smartphone has undoubtedly led the way here, providing researchers opportunities to be innovative in research design (Wellman, 2010). From their abundant everyday use, the dizzying array of platforms available through applications, their 'intuitive' accessibility and relatively reduced cost implications, smartphones have presented themselves as almost too tantalising not to deploy as innovative research tools (Birenboim and Shoval, 2016). Nevertheless, while mobile technologies may enliven contemporary qualitative research, questions can be raised regarding the moral and ethical implications and appropriateness of their use. Drawing upon empirical research carried out using a newly designed smartphone application, the remainder of this paper considers the moral implications of employing participants' personal technologies within research. First, we discuss the recent understandings of moral geographies and the smartphone as a geographical space. Next, after outlining the project from which this research has been conducted we explore morality as a conceptual lens through which to interpret the moral implications of accessing participants' digital 'spaces'. Finally, by way of concluding we offer insight into how researchers might consider more carefully the adoption of mobile technologies as research tools.

\section{Moral geographies}

To frame this discussion, we draw upon the 'moral turn' in Geography and how, since the latter part of the 1990s this has influenced consideration of ethics, care and responsibility. During this time attitudes towards social justice, radical politics, equitable policy directives and ethical academic practices were changing, being infused with what has become widely recognised as 'moral geographies' - the connections between places and behaviours and the appropriateness of expressing 
behaviours in and across space[s]. Moral geographies provide an excellent lens through which to examine these relationships between smartphones and research, particularly in terms of the people-place-technology connections afforded by their use. Matless (1994:127), for example examines the ways in which morality is conducted and received by people, in particular the "different ways of being in the world and the reactions of others to them". This blending of moral philosophical thought alongside geographical perspectives, while contentious, provides opportunities to 'think geographically' about the "spatial variations in everyday moralities [that] will inevitably be closely entangled with spatial variations in the 'structure' and 'functioning' of human groupings" (Philo, 1991, cited in Smith, $1997: 587)$. Pertinent to this study of using participants' smartphones in research is the notion that morality functions at various spatial scales. For example, Goffman (1963) examines the moralities involved in everyday interactions and social order, while Durkheim (1974) focuses on the ways in which morality can be internalised and embodied. This highlights the multidimensionality of morality whilst simultaneously emphasising individuals as being essentially "answerable only to oneself" (Foucault, 1988a:65).

Useful to extending this debate is McAuliffe's (2012) suggestion that moral geographies are concerned with how environments may be used and misused and how this encourages debates that question the appropriateness of particular/different behaviour[s] as well as the power dynamics that operate within spaces. Matless (1994), among others, employs Foucault's (1986) 'three senses of morality' to help disentangle people's ethical relationships to certain behaviours. This entails first outlining a moral code (essentially a set of rules) and interpreting the ways in which behaviours attached to the code may be considered obedient or transgressive, 
before reflecting upon how individuals relate to elements of the code as 'ethical subjects'. Foucault (1986:25) defines morality as "relat[ing] to the real behaviour of individuals in relation to the rules and values that are recommended to them", suggesting people's understandings of 'what is moral behaviour?' can be complex, diffuse and contested. Foucault's reasoning is useful to this investigation by raising questions about why research participants may appear to simultaneously acquiesce and/or resist elements of a research encounter, particularly when research is being conducted with[in] the ostensibly private sphere of a personal smartphone. This paper extends moral geographical work through attending to these asymmetric power relations that are evident when examining moral behaviours that are performed simultaneously in digital and geographical spaces, and questions whether these may be functions of hegemonic systems of 'acceptable' behaviour.

\section{Smartphone technologies}

This section examines the geographies of smartphones and how their usage creates unique opportunities and challenges for research. Smartphones have largely permeated contemporary lifestyles and are fundamental in shaping some of the activities many people undertake and the ways through which these activities are operationalised. Indeed, Lupton's (2013) discussion of the 'digital cyborg', a contemporary reworking of Haraway's cyborg concepts, highlights how technology has changed understandings of (and relationships with) bodies, and in a geographical sense, through the ways in which technology may extend the body, affecting bodily rhythms and interactions through space and time. Crucially, there is value in recognising how such 'technologies of the self' (Foucault, 1988b) are important devices for the creation and [re]definition of individual identities. Foucault's 
terminology is quite simply concerned with the methods through which individuals employ technologies that [re]create and [re]define the Self (Burkitt, 2002). Indeed, Kinsley (2014:371) describes the human/technological interface using the contexts of 'technics' as 'the problematic and constitutive relation between what we call 'human' and 'technology"' and relatedly "technicity' as the (emergent) qualities of that relation as it is performed". This demonstrates the symbiotic, or hybridised nature of smartphone usage - blurring the more typical boundaries between geographical and virtual space (de Souza e Silva, 2006). This was most recently witnessed in the summer of 2016 during the craze for the smartphone application 'Pokémon GO', a location-based game in which players explore the terrain of their local area in search of creatures contained within the application. This hybridisation of physical and digital space[s] using augmented reality demonstrates opportunities to explore new digital topographies and presents exciting ways for researchers to consider how humans might interact emotionally and affectively within their 'real' and 'imagined' surroundings.

Central to the success of smartphone technology has been the development of mobile applications (hereafter referred to as apps). Indeed, Wellman (2010:181) affectionately describes smartphones as "the Swiss Army knives of connectivity with cameras, GPS, compasses, and tens of thousands of other 'apps' [...] providing myriad affordances". Moreover, Christensen and Prax (2012) consider apps to be crucial to the networked relationships between people, mobilities, cultures, technologies and the internet. This essentially reconfigures assemblages of, and relationships between, people and practice (Goggin, 2009) by encouraging users to engage with spaces in novel and stimulating ways through the devices they hold in their hands. This opens up greater potential to examine new ways of understanding 
portability and connectivity in research, as well as how users might converge with one another or work through tasks in more novel ways (Laurier et al., 2016). Yet, as Goggin (2011) cautions, while smartphone technologies and apps may be heralded as a new cultural platform through which we might activate and disseminate knowledge, they are also relatively unsecured, unmediated, and untested as research devices. They carry risks of theft, damage and corruption (Welsh and France, 2012), as well as fears of disruption and other associated risks through accessing inappropriate content, misconduct and cyber-bullying, making them ethically and morally complex and problematic instruments to engage with in research. Hence, this paper postulates the risks involved in incorporating such shadowy technology uncritically into research design and how researchers may consider mitigating them.

\section{Researching with personal devices}

The research for this paper comes from a project conducted in 2016/17 in which we ${ }^{1}$ designed 'PlymTour' - a self-guided walking tour of Plymouth (UK), housed within a free and institutionally managed 'Mobile with Plymouth University' app that is available to Plymouth University students. This tour was designed to encourage students to access content about specific locations around the city (e.g. descriptions of locations, photographs, weblinks, quizzes etc.) directly from their smartphones whilst exploring Plymouth's city centre. In all, ten locations were included that comprised important touristic, historical, social and commercial spaces.

\footnotetext{
${ }^{1}$ This research team comprised two geographers who designed the content and a learning technologist from Plymouth University who coordinated the design of the app itself.
} 
Once the app had been developed, ethical approval was granted to conduct a pilot study with students recruited from the Geography department to examine how participants engaged with the app's interface, the locations (and routes between them) and content. We chose participant observation over other virtual/video ethnographies (see: Laurier et al., 2016; Duggan, 2017) as we were unable to secure permission to film in some of the private locations along the tour. Yet, by observing how our participants engaged with the technology, each other and the environment we ensured that our research remained 'in place' for the duration of the tours. To recruit willing participants we invited student groups undertaking a first year module that contained a field-based assessment that closely aligned with the content and locations we had developed to take part in the research. Information sheets were distributed to students during the first practical session for the module and groups were given the opportunity to contact us should they wish to take part. In all, four groups were involved, comprising seventeen students in total. These were selfselected, meaning group participants all knew each other, and there were mixed levels of knowledge of the technology and the locations. Prior to the research, participants were prompted to install or update their app and to ensure they had a full battery on their smartphones. Groups were asked to meet at one of the researchers' offices for a briefing on how to operate the app; to go through the structure and intentions of the research and complete consent forms; and for any questions the participants might have. Aside from health and safety guidance we were keen at this stage to not set out any rules or regulations. We wanted to facilitate a more 'natural' research environment that was about how the participants were interacting with the app, and not about how we might want them to use it or behave whilst using it. Each encounter took approximately $2.5-3$ hours to complete and we accompanied each 
group as they walked around the city, observing their use of the app, their interactions with one another and with the environment. Our observations were voice recorded and then later transcribed for analysis using Nvivo.

\section{[Re]appropriating technologies as 'research' spaces}

We begin by considering the spatial implications of incorporating personal devices in research. This came from warnings in the literature against re-appropriating social media, such as Facebook or Twitter, into spaces of research. Jarvis and Dickie (2010:177) argue that young people may consider their personal devices to be "invaded by academic materials" when asked to use them in learning or research situations. Pressures existed for us in interpreting how the students were engaging with the app, particularly as the participants' ownership of the technology, both materially and symbolically, meant we were not inclined to direct how they interacted with it. We wanted the participants to engage with it in the most 'natural' way possible without interference from us and in doing so we felt uncomfortable intervening if they appeared to disengage. This lead to a slight tension between a desire to better understand the students' use of the app and a hesitancy to question or intrude, as expressed in the researchers' observation below

"I think the phone use is note taking rather than app watching. That's one thing that's actually quite difficult with this approach without being able to intervene and ask. You don't want to peer over people's shoulders, it feels too rude!" (Group, C)

"So, one of them has got her phone in her hand, and I can see she's got the app open, but she's not looking at it. She's just, sort of, waving it around, and every so often glances at it." (Group A) 
This aligns with Thomas et al's. (2014) critique of employing personal technologies in learning situations. As these quotes imply, it is almost impossible to know fully how they are used in terms of what is viewed or what is shared, and our method of participant observation meant that we sometimes had to be very proximate to our participants to see what was going on. Yet what was interesting here was how our 'innovative' app was housed within the digital space of a smartphone that was very familiar to the participants. Hence the ubiquity of smartphone technology appeared to normalise their experience of the app, reducing its novelty and impact as both an educational and research tool.

Moreover, this complicates the moral roles of researcher and subject, and problematises our perceived identities as passive observers of these encounters. Instead we were positioned as active observers of a tool we had created and that was influencing our participants' engagements with their personal technologies (the presence of the app on their smartphones, our knowledge of the content of the app and the different ways that the participants might interact with it). Indeed, this forms what De Saulles and Horner (2011) have described as the 'portable panopticon', a digital version of Foucault's (1995) notions of surveillance whereby the smartphone becomes a space through which actions can be monitored and assessed. Here the smartphone becomes an apparatus of control through which behaviours are mediated virtually and anonymously. In the context of our research, we were, in many ways, privileged in knowing in advance how the app might shape the ways in which the participants moved through both the physical landscape of the city as well as the digital terrain of their private smartphone. Additionally, our status as observers meant we viewed the participants' use of the app from a distance, essentially reducing them to being "the object of information, [but not the] subject in 
communication" (Foucault, 1995:200). Yet while this positionality was unintentionally performed, it is crucial to recognise that these power dynamics have consequences when using participants' personal devices in research. In developing this we acknowledge McAuliffe's (2012:203) suggestion to recognise a "politics of place" when appropriating space, particularly in terms of how we might interpret different behaviours that are expressed using digital devices. We therefore identify the value in balancing engagement with research with being sensitive to the ownership of other private spaces within personal technologies that help produce behaviours that fit within how participants (and researchers) perceive acceptable behaviours and assumed moral rules.

\section{The merging of habits and identities in smartphone spaces}

To further understand these implications for researching with participants' digital technologies we draw upon Foucault's (1986) interpretation of morality and practices of the Self to gauge how and why the participants might have interacted differently with their smartphones once they had been asked to use them in the research. Foucault highlights the ambiguity of morality as a word, specifically in terms of the hierarchical and potentially hegemonic sets of values and rules that may constitute 'moral behaviours'. Here we concern ourselves with morality in terms of principles or rules of 'correct' conduct - put simply, the distinction between right and wrong - that may be assumed or implied by individuals. Foucault problematises this though by suggesting that individuals' moral behaviours (or codes) are complexly interrelated being constantly corrected, counterbalanced and cancelled out depending upon how individuals engage with certain situations in space and time. During our research encounters, interpreting these behaviours became complicated as there were 
inconsistencies in how participants engaged with devices and spaces both across the groups and for the duration of the tours:

"I think one person has got their phone out but I think they're probably taking notes or on Facebook, rather than actually using it to look at the app." (Group D)

"One person has got their phone out but it looks like they're looking at other things on the phone rather than actually using it to look at the app." (Group B)

These comments indicate how the ways in which the participants conducted themselves in relation to what Foucault (1986) refers to as the prescriptive elements of a moral code was important. We refrained from asking participants not to use their smartphones for social media during the research yet, as the quotes imply, this raised a dilemma over what might be deemed the 'appropriate usage' of a personal device as a research instrument (e.g. as this is a research encounter should the PlymTour app take priority over social media for the duration of the research?). Mediating this is, of course, an impossible (and impractical) task, particularly as the participants were utilising a device they had significant identity connections with, meaning expecting them not to continue expressing their habits whilst using their smartphones could be considered unreasonable.

This raises questions of what might be considered the ethics of changing behaviours in different socio-spatial and public/private spaces (Smith, 1997). This is pertinent as moral behaviours can be viewed as processual, in that they are incorporated into everyday practices (Giddens, 1991). Bourdieu's (1990) theories of habitus and capital are useful here in revealing links between disposition and practice and the disturbance of routines. While Bourdieu does not attend explicitly to notions of ethics 
and morality in his research, his understandings of Self and Other certainly provide a foundation from which to examine moral judgements. Drawing on Bourdieu's ideas of the field, Lamont (1992), for example, examines the 'moral boundaries' that may be erected to delineate acceptable behaviours in certain social fields. This identifies complexities regarding the roles of power, mobility and structure in determining how and why 'moral signals' are produced as well as the vital resource they provide in establishing different social positions. Ignatow (2009) extends this by suggesting that moral behaviours (in this case, the use of a personal device as part of a research encounter) are a product of embodied and cognitive practices that are then practiced (and perhaps mediated) in the realm of negotiated social discourses (here, by both researcher and participant). Hence, we recognise that research using personal technologies does not exist in a spatial, or temporal vacuum, meaning participants cannot (and should not) divorce themselves from their digital worlds when participating in research. Indeed, while Laurier et al. (2016) argue that smartphones have reconfigured our understandings of place and mobility through mediation between habit and reflexivity, our research suggests that this demonstrates the pliability, permeability and flexibility of digital moral boundaries, particularly in terms of how these are then overlaid onto geographical spaces.

\section{Resistance, disengagement and disruption}

The final theme is concerned with the subtle forms of resistance, disengagement and disruption that were evident in our research. McAuliffe (2012:191) draws upon Cresswell's work on transgressive people, behaviours and practices that are deemed 'out of place' in mainstream society, arguing that: 
"Moral geographies reflect the normative expectations of the use and misuse of particular environments, and as such can form the basis of inclusion and exclusion as the transgression of normal ways of being in a place are policed in order to ensure the maintenance of the common good."

Yet, defining 'normal ways of being' can be a thorny topic, particularly as 'our' moralities as researchers inevitably differed from those of our participants. For example, Goffman (1963:8) explores the influence of different behaviours upon social systems by considering 'social order' to be formed through "the consequence of any set of moral norms that regulates the way in which persons pursue objectives". Indeed, Giddens (1991) suggests that to normalise moral orderings is to consider behaviour to be bound in traditional practices that resist 'new' practices, meaning deviations from tradition (or what may be perceived to be traditional) may cause consternation. This was evident in our study and, as the following excerpts attest, while these examples were not instances of bad behaviour, they illustrate a subversion of what might be assumed codes of behaviour in research practice:

"Okay, [some students are] taking selfies [around stop one]. I think that's probably quite interesting in terms of safety and comfort, for the students to have their phones out and feel comfortable with doing that sort of thing." (Group D)

"We're on Union Street and some of the boys are larking around rattling doors on the derelict Palace Theatre and trying to take photos through the smashed windows. Two guys have just walked past us and were taking the mickey [but] I don't think anyone noticed that actually. I don't think the students noticed." (Group C) 
What is important to recognise here then is that moral codes are relational, in terms of "what actually is right or wrong differs among individuals, societies and cultures" (Smith, 1997:586). Yet, as the activities in the quotes suggest, this relationality makes moral behaviour difficult to distinguish. Durkheim (1974) argues that from an individualistic perspective, morality is measured internally, rather than externally, rendering appraising moralities (e.g. intentions, conduct and accountabilities) an act of the Self and not of the Other. Hence, individuals submit voluntarily to that moral code rather than by force. This resonates with our encounters (e.g. the taking of selfies and the hijinks that reflected the participants' relative aged identities and practices), particularly as the moral codes at play during our research appeared opaque and existed in a state of flux, meaning they were difficult to discern, let alone define.

In reconciling this, by utilising personal smartphones for research purposes we were bound to invite playful transgressions alongside opportunities for self-reflexivity and enlightenment, yet by avoiding mediation - and avoiding overlaying our own moral codes - we encouraged the participants to draw (and re-draw) their own moral boundaries in ways that supported both our research needs and the participants' routinized behaviours. Hence, we were mindful of the implications for what could be assumed to be 'appropriate behaviours' during the research encounters (and if these could be assumed to be [in]appropriate at all!). To support this, Butler (2005) advises being reflexive and critical of the changing social contexts through which interpretations of morality emerge, meaning while moralities are produced and negotiated through socio-historical contexts these are not fixed and can be adapted or reconciled depending on their situation. For example, we were aware of the potential impact that our predefined positions of lecturer/student may have brought to 
these encounters (e.g. the assumption that smartphone use is disruptive in learning situations, or that 'teachers' might discipline inappropriate uses of technology (Thomas et al., 2014)) and the hierarchies and power imbalances that these may constitute. We were therefore keen to avoid turning these encounters into 'mobile classrooms' with codified rules about how/when to use smartphones. Beyond providing information on health and safety and briefing the students on privacy and data protection we were conscious to play down any sort of code of conduct or lecturing on 'appropriate' interactions with us as researchers.

Notwithstanding, despite our attempts to minimise guidelines of 'correct' behaviours, we were still complicit in the production of our participants as, what Foucault (1986) terms 'ethical subjects'. In doing so we were keen to provide the participants greater agency in developing their own senses of what ethical behaviours might look like with/in technological spheres to encourage them to more readily consider their own moral responsibilities (e.g. allowing leadership and teamwork to emerge and be managed or letting participants decide how and when they might engage with the app). So, by not privileging our research we allowed for playfulness and agency to permeate the encounters, encouraging sensitivity to the "different ways of being in the world and the reactions of others to them" (Matless, 1994:127). As such, rather than penalising infractions of assumed rules and conduct, the diverse range of behaviours became part of the research through the ways in which the participants formed their interactions with the technology, the environment and with each other.

\section{Conclusion}

In conclusion, this paper began by outlining the ways in which mobile technologies have transformed understandings of, and relationships with, bodies and 
digital/geographical spaces and how this might add value to self-directed research or research with others. Yet, while it is agreed that this may generate new ways of understanding mobility, connectivity and the convergence of subjects in research, we have been critical of mobile digital technologies being considered an unproblematic addition to the qualitative social research toolkit. By drawing upon notions of morality we sought to tease out the entangled moral, ethical and spatial implications connected to utilising participants' personal devices as instruments of research, and from this we can offer three key recommendations that recognise the complexities of researching with[in] participants' smartphones.

We first acknowledge the risks involved in trespassing into participants' digital spaces, stressing the importance of not underestimating the position of the researcher and subject during research encounters. While institutions may grant ethical approval, and participants may provide informed consent to conduct research using personal technologies, these spaces are voluminous and complexly interconnected with one another. Notwithstanding, in extending Jarvis and Dickie (2010) this should not necessarily mean that such spaces are off-limits. By examining the politics of place (McAuliffe, 2012) present in research encounters we advocate recognising participants' material and symbolic ownership of their technologies as influenced by the moral boundaries that they may erect and/or dismantle and how this contributes towards shaping, and reshaping, the social positioning of researchers and subjects during research encounters.

Expanding on this, we support claims that personal technologies can be tools that represent participants' habitual identities and are thus influenced by their individual, and complexly interrelated, moral codes. By drawing upon Foucault's (1986) 
interpretation of the Self as a non-prescriptive and constantly evolving entity that continually [re]negotiates the relationship between Self and Other, we argue against privileging research over access to non-academic digital spaces. The boundaries between 'good' and 'bad' digital behaviour are decidedly opaque and should be approached with caution. Expecting participants not to slip into digital habits (checking social media, taking photographs, accessing different apps/content etc.) is unreasonable as this is simply behaviour that falls within the remit of their 'normal' moral identities, as opposed to a research encounter, which is decidedly extraordinary.

Our third recommendation draws upon the subtle subversive and disruptive behaviours that were witnessed during our research encounters. By examining this through the lens of morality we considered these subversions not as infractions on morality, but as a method of articulating alternative interpretations of morality that expressed agency, playfulness and reactivity. Here, we argue that researchers could reconsider normative representations of research fields as spaces to acquiesce by contemplating the value of participants 'misusing' research spaces (both 'real' and 'virtual') in order to assist in smoothing power irregularities between researchers and participants that may potentially [re]invigorate the research encounter in ways that have not necessarily been considered.

Finally, in drawing these themes together, we contribute towards discussions of moral geographies by identifying how the irregular power dimensions involved in moral behaviours may be functions of hegemonic systems of 'acceptable' behaviour - particularly in terms of how moralities are expressed and understood. To borrow from Laurier et al. (2016), our research examines how group intersubjectivities may 
[re]produce certain behaviours during research encounters that shape, and are shaped by, different person-place-technology assemblages.

\section{References}

Birenboim A and Shoval N 2016 Mobility research in the age of the smartphone Annals of the American Association of Geographers 106 283-291

Bourdieu P 1990 The logic of practice Cambridge UK, Polity Press

Brown B McGregor M and Laurier E 2013 iPhone in vivo: video analysis of mobile device use. Proceedings of the SIGCHI conference on Human Factors in computing systems 1031-1040.

Burkitt I 2002 Technologies of the self: habitus and capacities Journal for the Theory of Social Behaviour 32 219-237

Butler J 2005 Giving an account of oneself. Oxford, Oxford University Press.

Christensen C and Prax P 2012 Assemblage, adaptation and apps: smartphones and mobile gaming Continuum 26 731-739

De Saulles M and Horner DS 2011 The portable panopticon: morality and mobile technologies Journal of Information, Communication and Ethics in Society 9 206-216

De Souza e Silva A 2006 From cyber to hybrid: mobile technologies as interfaces of hybrid spaces Space and culture 9 261-278

DeLyser D and Sui D 2013 Crossing the qualitative-quantitative divide II: inventive approaches to big data, mobile methods, and rhythmanalysis Progress in Human Geography 37 293-305 
Duggan M 2017 Questioning "digital ethnography" in an era of ubiquitous computing Geography Compass 111-12.

Durkheim E 1974 Sociology and philosophy. New York, Simon and Schuster

Foucault M 1986 The use of pleasure: the history of sexuality, vol. 2 London, Penguin

Foucault M 1988a The care of the Self: the history of sexuality, vol 3 London, Penguin

Foucault M 1988b Technologies of the self in Martin LH Gutman H and Hutton PH eds Technologies of the self: a seminar with Michel Foucault Cambridge, Mass., MIT Press 16-49

Foucault M 1995 Discipline and punish: the birth of the prison New York, Vintage Books

Goffman E 1963 Behavior in public places New York, Simon and Schuster

Goggin G 2009 Adapting the mobile phone: the iPhone and its consumption Continuum 23 231-44

Goggin G 2011 Ubiquitous apps: politics of openness in global mobile cultures Digital Creativity 22 148-159

Gorman R 2017 Changing ethnographic mediums: the place-based contingency of smartphones and scratchnotes Area 49 223-229

Ignatow G 2009 Why the sociology of morality needs Bourdieu's habitus Sociological Inquiry 79 98-114 
Jarvis C and Dickie $\mathbf{J} 2010$ Podcasts in support of experiential field learning Journal of Geography in Higher Education 34 173-186

Kinsley S 2014 The matter of 'virtual' geographies Progress in Human Geography 38 364-384.

Lamont, M 1992 Money, morals, \& manners: the culture of the French and American upper-middle class Chicago, University of Chicago Press

Laurier E Brown B and McGregor M 2016 Mediated pedestrian mobility: walking and the map app Mobilities 11117-134.

Lupton D 2013 The digital cyborg assemblage: Haraway's cyborg theory and the new digital health technologies in Collyer $\mathbf{F}$ ed The handbook of social theory for the sociology of health and medicine Houndmills, Palgrave Macmillan 567-581

Matless D 1994 Moral geography in Broadland Ecumene 1 127-155

Matless D 1997 Moral geographies of English landscape Landscape research 22 $141-155$

McAuliffe C 2012 Graffiti or street art? Negotiating the moral geographies of the creative city Journal of urban affairs 34 189-206

Neff G and Nafus D 2016 Self-Tracking Cambridge, MIT Press.

Smith DM 1997 Geography and ethics: a moral turn? Progress in Human Geography 21 583-590 
Thomas KM O'Bannon BW and Britt VG 2014 Standing in the schoolhouse door: teacher perceptions of mobile phones in the classroom Journal of Research on Technology in education 46 373-395

Wellman B 2010 The reconstruction of space and time: mobile communication practices Contemporary Sociology: A Journal of Reviews 39 179-81

Welsh K and France D 2012 Spotlight on...smartphones and fieldwork Geography $9747-51$. 